\title{
Learning high level programming in a virtual laboratory using the SimulNet educational platform
}

\author{
Pedro Rodríguez, Alfonso Fernández, Luis Anido, Juan Santos, Judith \\ Rodríguez and Manuel Caeiro \\ Departamento Ingeniería Telemática, ETSE Telecomunicación, University of Vigo, Spain
}

\begin{abstract}
One of the weakest fields in WWW-based education is practical training, which is carried out in teaching laboratories in conventional education. SimulNet is a simulation-based platform for virtual laboratories on the internet, that supports interactive and collaborative practical training. Over the past few years, some simulators have been developed for the SimulNet platform focused on the area of computer architecture. Now, we present a Pascal simulator that provides an environment where learners can experience high level programming concepts in the same way they can when using compilers like Turbo Pascal or Free Pascal. In this way, learners are provided with the same programming facilities of an integrated development environment but in a virtual laboratory environment, where communication, collaboration and supervision are possible.
\end{abstract}

Keywords: virtual laboratories, web-based training, computer training

\section{INTRODUCTION}

Advances in information and communication technologies, and specifically in multimedia, networking, and software engineering promote the appearance of a new generation of computer-based training systems. The internet is today a ubiquitous supporting environment for virtual and distributed learning environments. As a consequence, many institutions, both public and private, take advantage of new technologies to offer training products and services at all levels using the world wide web.

This paper focuses on the use of the internet as an environment for telelearning and specifically on practical training resembling traditional teaching laboratories. Section 2 is devoted to the presentation of SimulNet, an

The original version of this chapter was revised: The copyright line was incorrect. This has been corrected. The Erratum to this chapter is available at DOI: 10.1007/978-0-387-35615-0_52 
educational platform whose aim is to provide a virtual laboratory environment where simulators of conventional laboratory tools can be introduced to put theoretical knowledge into practice. SimulNet provides common services and facilities, like communication and collaboration tools, tutoring services, and other value-added services, that are directly included in the simulators incorporated into the platform.

Following this idea, we have developed a new simulator devoted to Pascal programming. It supports the development of Pascal programs and applications in the same way as traditional tools like Turbo Pascal (Hennefield) or Free Pascal compiler (web-site). The prime area of application that Pascal supports is the learning environment. In fact, it was developed to be used for teaching students the basics of programming in a high level language. Since the early 1970 s to the late 1990s Pascal was the language of choice for teaching programming techniques. The Pascal simulator is described in Section 3. We will finish by drawing some conclusions in Section 4.

\section{SIMULNET: SIMULATORS OVER THE NETWORK}

SimulNet (Llamas, Anido and Fernandez, 2001) is a simulation-based platform for virtual laboratories on the internet. Unlike other CBT systems whose aim is to achieve a virtual classroom, SimulNet provides a virtual laboratory to put theoretical knowledge into practice. To achieve this goal, distributed applications that are simulators of those tools and that can be found in a conventional laboratory need to be developed and embedded in the SimulNet platform.

SimulNet provides simulator developers with a specific API to take advantage of the whole platform functionality. SimulNet developers only have to worry about the simulator itself. It must reflect the behaviour of the system as closely as possible to the real one. Connection to the system, authentication, monitoring and co-operative learning are provided by the SimulNet API.

This labware can be delivered via the web or by CD-ROM. In the former, it provides a full virtual tele-laboratory atmosphere, including co-operative learning with other students and tutoring facilities for teachers. In the latter, it allows stand-alone learning and, at the same time, students can connect to the server side and benefit from the tele-laboratory facilities. 


\subsection{Architecture}

The implementation of SimulNet is based on internet technologies which are currently available, especially Java. Thanks to Java technology we have achieved several important features in SimulNet:

- Platform independence. Client and server applications may be run on any computer whatever its operating system or architecture.

- Java eases the development of WWW-based applications. SimulNet provides a WWW client (based on Java applets) and a stand-alone client (based on Java applications) delivered by CD-ROM.

- The simulators are interactive. There is no network overhead as the simulators run on the student's own computer.

Users access SimulNet using a standard WWW browser, which presents an HTML document provided by the HTTP server on the server side (see Figure 1). This document contains an embedded Java applet that would start and stop any other Java application provided on the server side through the internet. In this way, no additional software is required apart from the browser itself.

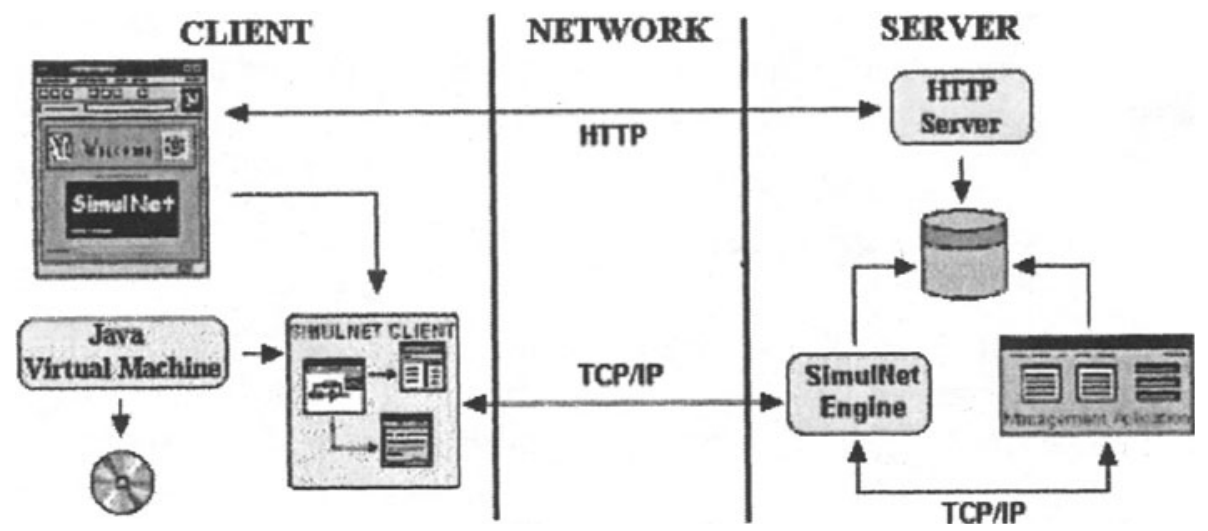

Figure 1. Pascal simulator structural model

The server side functionality is implemented by three different applications. The first one is the WWW server whose function is to provide HTML documents containing the SimulNet applets. Communication with the WWW server is based on HTTP protocol. The SimulNet management application carries management functionality and simulator configuration for every trainee and tutor. Finally, the SimulNet engine is the Java application that supports the functionality of our system. It implements the system's behaviour and is set up using the management application. 


\subsection{Collaborative learning and tutoring facilities}

SimulNet provides several communication tools to support collaborative learning (a Talk Tool, Whiteboard Tool, Bulletin Board, Mail Tool, Multitalk Tool, and Project Management Tool). All of them were developed from scratch, and they can be easily included in new simulators thanks to the SimulNet API.

In this way, students are provided with an easy-to-use set of collaborative facilities to ease learning and practical training. There is no need to set up or use additional software at client computers, which may prove to be difficult for trainees. The software needed is automatically downloaded from the network and can be run wherever the user might be, regardless of the particular hardware platform or operating system.

In addition to the collaborative facilities, SimulNet provides a monitoring system to follow the students' interaction with the system on-line, or off-line. In any case, it is necessary to define the user actions that will be considered for tracking. These actions will be reflected in students' traces. Whenever a student performs a given task, a report is sent to the tutor responsible for monitoring his or her actions. Furthermore, teachers are allowed to undertake 'off-line' monitoring. Since the students' traces are stored on the SimulNet server, teachers are able to supervise previous trainees' learning sessions to check that students have succeeded in their training practices.

\section{THE PASCAL SIMULATOR}

The Pascal simulator, included as a SimulNet simulator, is intended for learning and training the basics of programming in a high level language. The prime area of application that Pascal entails is the learning environment. Learning Pascal requires training support, so learners can experience the language elements explained in theoretical lectures.

\subsection{Development and structure}

The Pascal compiler has been developed by focusing in on the logic needed to provide the functionality related to Pascal program editing, compiling and execution. The SimulNet services and facilities are embedded in the same way as that in other simulators. 


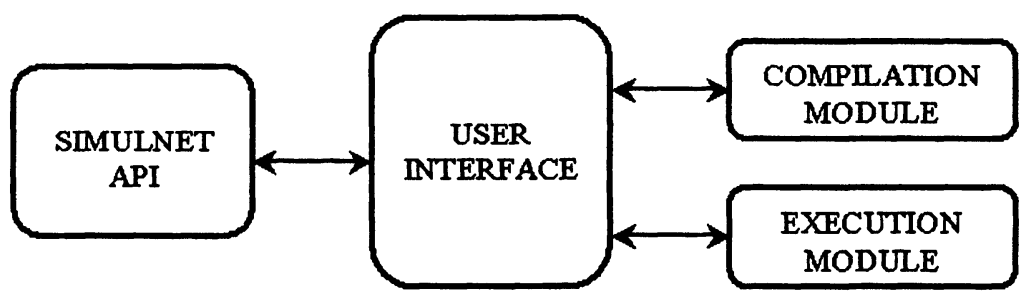

Figure 2. Pascal simulator architecture

Figure 2 depicts the architecture of the Pascal simulator. Specific business logic is related to the simulation of Pascal program compilation and execution. These tasks are carried out in two specific modules that were developed following guidelines from the compiling bibliography (Aho, Sethi and Ullman, 1985; Levine, Mason and Brown, 1995; Sun Micro systems web-site, 2001).

\subsubsection{Compilation module}

This module is in charge of simulating the compilation of a Pascal source program. The program compilation includes two clearly separate stages:

- The first stage is devoted to the analysis of the program. A Java parser (a syntax and lexical analyser) specific for Pascal was included, with the purpose of identifying the lexical components of Pascal and the grammatical combinations of them.

- The second stage is devoted to the creation of a set of data structures, which are needed to perform the simulation of the program execution to test types, ranges, etc. in preparing the program to be simulated. Unlike true compilers, binary code is not generated at this stage.

\subsubsection{Execution module}

This module allows the simulation environment to execute a Pascal program. As Java applets are not allowed to access the user computer memory, we need to simulate Pascal program execution.

The execution module contains a parser, similar to that of the compilation module, which creates additional data structures needed to simulate the execution of a Pascal program. In addition, this module supports a standard input/output console where the user can see the messages that his/her program generates, and where he/she can introduce any requested data. 


\subsubsection{The SimulNet API}

The SimulNet platform provides a Java API (Llamas, Anido and Frenandez, 2001) to enable the introduction of the platform services and facilities into new simulators. Programmers should import some packages, implement some interfaces and use some methods whenever a SimulNet facility is required.

For example, what we have to do to connect the Pascal simulator (both Java applet or Java application) to the SimulNet Engine is to create an object of the class simulnet.connection.AgentConnection with the following parameters: host and port of the server, user's login and type of user (teacher/student). Afterwards, we only have to invoke a method. After the successful connection to the server side, we are allowed to create the main object at the client side: MyModule = new CommunicationsModule (socket,user,language); where language indicates the language chosen for the user interface (English, Spanish or Galician). At this point, we are ready to use communication tools or to monitor students.

\subsubsection{User interface module}

The user interface deals with all the functions and services available for the user. They include the specific Pascal functions, related to program editing, compilation and execution, and the services and facilities provided by the SimulNet Platform.

Figure 3 shows the Pascal simulator user interface. The interface consists of a menu bar, a button bar, an editor, and a status bar. In addition, an input/output console is available, and several windows may appear to show messages and warnings. The user interface is available in three different languages: English, Spanish and Galician. New languages can be supported easily through the introduction of the corresponding mappings.

\subsection{Pascal features and elements supported}

The purpose behind the development of the Pascal simulator was to provide a programming tool similar to Turbo Pascal 7.0 version. Turbo Pascal gives an easy-to-use integrated development environment (IDE) for complete access to a broad set of tools, that make it possible to edit, compile, run, and debug programs from an interconnected environment. That is why our goal was to support the Turbo Pascal 7.0 version. Using our simulator, learners can edit, compile and run their Pascal programs, and they can use the Turbo Pascal 7.0 (Borland) reference books. 
The Pascal simulator supports almost all of the elements available in Turbo Pascal 7.0. But in some cases, Java restrictions force us to adopt different solutions. For example, it is not possible to make any direct reference to memory, because the data structure of the Pascal program is emulated by the simulator and it has a logical organisation in Java objects not related to any physical organisation of the memory. Another important restriction is related to file management and processing. While text files are completely compatible with conventional ones, our data files maintain a different format, because they present a Java-specific byte code.

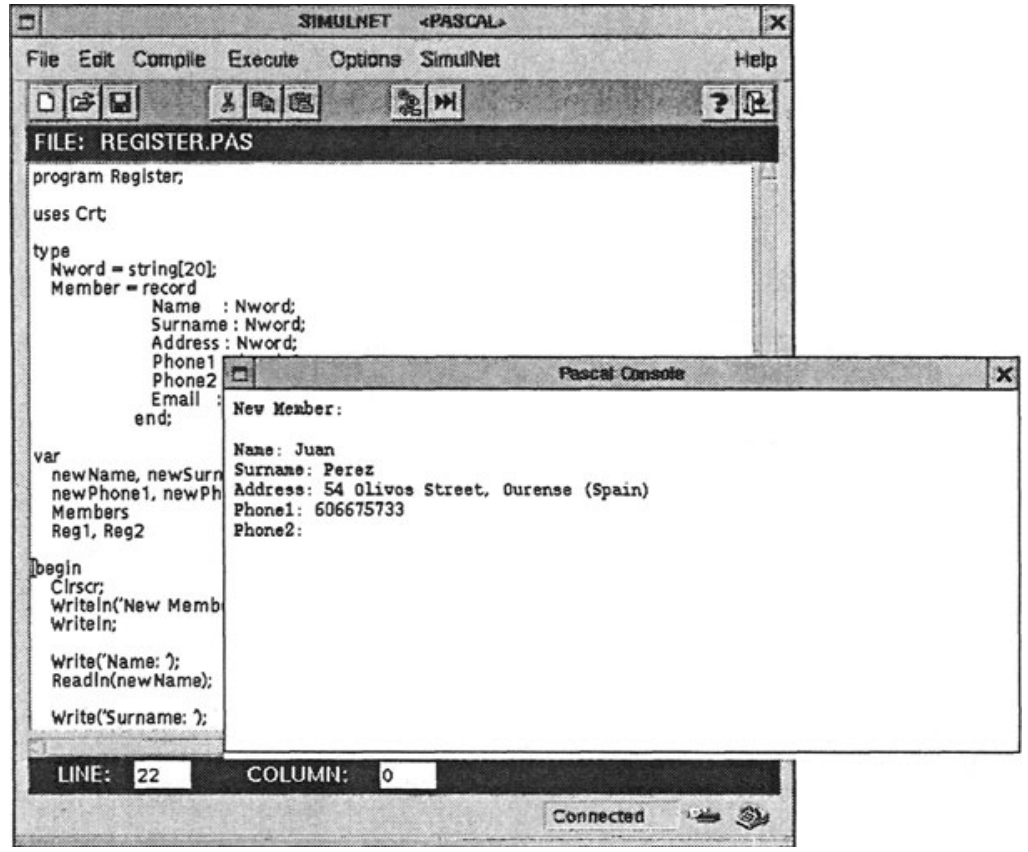

Figure 3. Pascal simulator interface

\section{CONCLUSIONS}

The system described allows students to gain expertise in high level programming. Obviously, the simulator has several limitations which we will try to solve in the near future. We have developed some IDE environments devoted to computational science and low level programming training (Llamas, Anido and Fernandez, 2001) that have been proved as useful tools. 
This simulator is supported by SimulNet, a platform whose main aim is to deliver software through the internet or by CD-ROM technology. The technology used to develop SimulNet allows us to forget about different hardware platforms or operating systems thanks to the "write once, run anywhere" Java philosophy. The administrative and maintenance tasks are straightforward, and collaborative and co-operative training is immediately supported thanks to the communication and tutoring services provided.

\section{REFERENCES}

Aho, A.V., Sethi, R. and Ullman, J.D. (1985) Compilers: Principles, Techniques, and Tools. Boston, MA: Addison-Wesley

Borland International Inc. Turbo Pascal 7.0. Language Guide, Turbo Pascal 7.0. User Guide, and Turbo Pascal 7.0. Programmer Reference

Hennefeld, J. Using Turbo Pascal 6.0-7.0. Boston, MA: PWS Publishing Company, Free Pascal Compiler. Available at http://www.freepascal.org/ (last accessed November 28th, 2001)

Levine, J.R., Mason, R. and Brown, D. (1995) Lex and Yacc. Second Edition. Cambridge, MA: O'Reilly and Associates, Inc.

Llamas, M., Anido, L. and Fernandez, M.J. (2001) Simulators over the Network. IEEE Transactions on Education, 44, 2 ,

Sun Java Official Web Site. Available at http://java.sun.com (last accessed November 28th, 2001)

Sun Microsystems. JavaCC Documentation. Web site at http://www.metamata.com/javacc (last accessed November 28th, 2001)

\section{BIOGRAPHIES}

Pedro Rodríguez received a telecommunication engineering degree from the Technical University of Madrid in 1989, and a doctorate in telecommunications (in 1998) from the University of Vigo, Spain. In addition to teaching, he is a member of the GIST group involved in research in the areas of real-time systems. Alfonso Fernández is a final year telecommunication student. The other authors are teachers and members of the GIST research group at the University of Vigo. 